\title{
1 Automatic and fast encoding of representational uncertainty underlies probability distortion
}

$$
\text { Xiangjuan Ren }{ }^{1,2,3} \text { Huan Luo }{ }^{1,4,5 *} \text { Hang Zhang }{ }^{1,3,4,5 *}
$$

1. School of Psychological and Cognitive Sciences and Beijing Key Laboratory of

$$
\text { Behavior and Mental Health, Peking University, Beijing, China }
$$

*Corresponding authors:

Huan Luo, email: huan.luo@pku.edu.cn

Hang Zhang, email: hang.zhang@pku.edu.cn 


\section{Abstract}

23 Humans do not have an accurate representation of probability information in the

24 environment but distort it in a surprisingly stereotyped way ("probability distortion"), as

25 shown in a wide range of judgment and decision-making tasks. Many theories

26 hypothesize that humans automatically compensate for the uncertainty inherent in

27 probability information ("representational uncertainty") and probability distortion is a

28 consequence of uncertainty compensation. Here we examined whether and how the

29 representational uncertainty of probability is quantified in the human brain and its

30 relevance to probability distortion behavior. Human subjects kept tracking the relative

31 frequency of one color of dot in a sequence of dot arrays while their brain activity was

32 recorded by magnetoencephalography (MEG). We found converging evidence from

33 both neural entrainment and time-resolved decoding analysis that a mathematically-

34 derived measure of representational uncertainty is automatically computed in the brain,

35 despite it is not explicitly required by the task. In particular, the encodings of relative

36 frequency and its representational uncertainty respectively occur at latencies of

37 approximately $300 \mathrm{~ms}$ and $400 \mathrm{~ms}$. The relative strength of the brain responses to

38 these two quantities correlates with the probability distortion behavior. The automatic

39 and fast encoding of the representational uncertainty provides neural basis for the

40 uncertainty compensation hypothesis of probability distortion. More generally, since

41 representational uncertainty is closely related to confidence estimation, our findings

42 exemplify how confidence might emerge prior to perceptual judgment. 


\section{Introduction}

44 Humans do not have an accurate representation of probability or relative-frequency

45 information in the environment but distort it in a surprisingly stereotyped way. Typically,

46 small probability is overestimated and large probability underestimated, which can be

47 well fit by a linear-in-log-odds (LLO) model with two parameters (see [1] for a review).

48 Such "probability distortion" phenomena occur in a variety of judgment and decision-

49 making tasks such as relative-frequency estimation [2-4], confidence rating [5-7],

50 decision under risk [8-11], and are also widely reported in animal behaviors [12-14].

51 However, the neural computations that accompany such probability distortions remain

52 largely unknown.

53 One computation that is assumed to be central to probability distortion [9, 15-17]

54 is compensation for the uncertainty inherent in the representation of noisy probability

55 information (“representational uncertainty"). In particular, recent comparison among an

56 extensive set of computational models of probability distortion [9] suggests that in their

57 judgment and decision-making people take into account representational uncertainty

58 that is proportional to $p(1-p)$, where $p$ denotes probability or relative frequency. That

59 is, representational uncertainty is zero at $p=0$ or 1 and maximal at $p=0.5$. The $p(1-$

$60 p)$ form had also been proved by Lebreton et al. [18] as a measure of uncertainty that

61 correlates with the explicitly reported confidence for a specific judged value.

62 Specifically, their fMRI results showed that during the valuation process, this

63 uncertainty measure is automatically encoded in the ventromedial prefrontal cortex

64 (vmPFC) - the brain region known for encoding value-even in the absence of explicit 
confidence rating. Motivated by previous neuroimaging studies and computational modelling, we ask whether representational uncertainty of probability information can be automatically encoded in human brain and if yes, whether this encoding can precede the explicit judgment of probability, as theories of probability distortion would expect $[9,15-17]$.

In the present study, we designed a new experimental paradigm where a major component of the representational uncertainty of probability information (i.e. $p(1-p)$, see Results for details) was varied continuously over time. By using time-resolved neural measurements and assessing the temporal coupling between stimulus variables and ongoing brain activities, we examined whether and how the encoding of representational uncertainty proceeds in time and relates to probability distortion.

Twenty-two human subjects participated in the study and were instructed to continuously track the relative-frequency $(p)$ of one color of dots in a sequence of dot arrays (Fig 1A), during which their brain activities were recorded by magnetoencephalography (MEG). First, we found that, though $p$ was the only variable that subjects needed to track while $p(1-p)$ was task-irrelevant, the periodic changes of $p(1-p)$ as well as $p$ entrained neural rhythms, supporting an automatic tracking of representational uncertainty in the brain even when it is task-relevant. Next, by using a time-resolved decoding analysis to delineate the temporal course, we further found that the encoding of $p$ and $p(1-p)$ peaked around 300 and $400 \mathrm{~ms}$, respectively. Finally, the relative strength of the neural responses to the two variables ( $p$ and $p(1-p))$ in the parietal region correlated with the variation of probability distortion behavior across 
bioRxiv preprint doi: https://doi.org/10.1101/2019.12.17.879684; this version posted June 1, 2020. The copyright holder for this preprint (which was not certified by peer review) is the author/funder, who has granted bioRxiv a license to display the preprint in perpetuity. It is made available under aCC-BY 4.0 International license.

87 individuals. Taken together, our results provide neural evidence for an automatic, fast

88 encoding of representational uncertainty in the human brain that might underlie

89 probability distortion observed in a wide range of human behaviors.

90

91 


\section{Results}

93 As shown in Fig 1A, on each trial, subjects saw a sequence of displays consisting of

94 isoluminant orange and blue dots that was refreshed every $150 \mathrm{~ms}$, and were

95 instructed to track the relative-frequency of dots in one color (i.e. orange or blue,

96 counterbalanced across subjects). To ensure subjects' persistent tracking throughout

97 the trial, the sequence of displays ended after random duration and subjects needed

98 to report the relative-frequency of the last display by clicking on a percentage scale

99 afterwards.

A

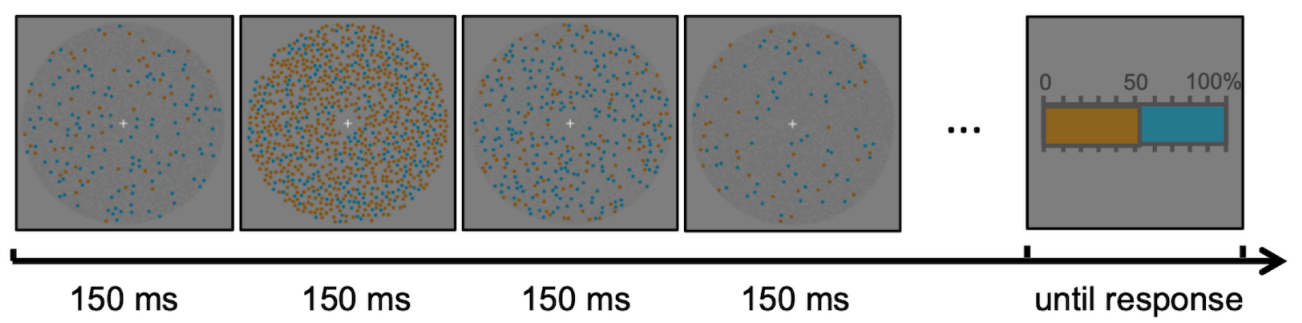

B

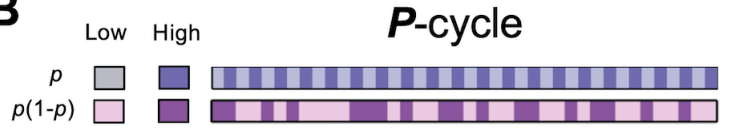

101

102

103

104

105

106

107

108

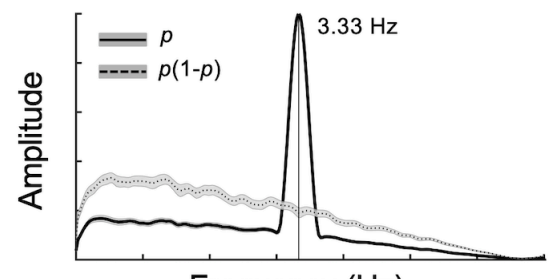

Frequency $(\mathrm{Hz})$
Fig 1. Experimental design and behavioral results.

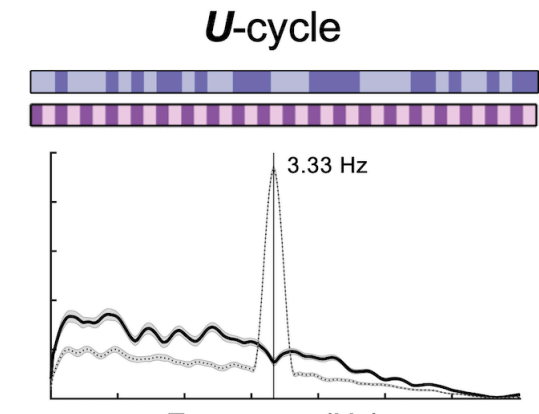

Frequency $(\mathrm{Hz})$

(A) Relative-frequency tracking and judgment task. On each trial, a sequence of orange and blue dot arrays was presented at a rate of $150 \mathrm{~ms}$ per display. Subjects were asked to fixate on the central fixation cross and track the relative frequency of orange (or blue) dots in each display until a $0-100 \%$ response scale appeared. Subjects then clicked on the scale to report the relative frequency in the last display. (B) The $p$ and $p(1-p)$ sequences in an example $P$-cycle or $U$-cycle trial. The value of $p$ on each display was randomly chosen from a uniform distribution ranging from 0.1 to 0.9 . In $P$-cycles (left 
panels), the $p$ sequence was generated in such a way that the $p$ value in different displays alternated between lower $(<0.5)$ and higher $(>0.5)$ values, resulting in a frequency spectrum peaking at $3.33 \mathrm{~Hz}$ (solid line), while the corresponding $p(1-p)$ sequence was aperiodic (dashed line). In $U$-cycles (right panels), it was the reverse: the $p(1-p)$ sequence alternated between lower $(<0.21)$ and higher $(>0.21)$ values in cycles of $3.33 \mathrm{~Hz}$ (dashed line), while the $p$ sequence was aperiodic (solid line). To illustrate the periodicity or aperiodicity in the $p$ and $p(1-p)$ sequences, the lower and higher $p$ values, and lower and higher $p(1-p)$ values are coded in different discrete colors in the bars above the frequency spectrum plots. Note that this discretization is only for illustration purposes and all data analyses were still based on continuous values of $p$ or $p(1-p)$.

A 2 ( $P$-cycle or $U$-cycle) by 2 ( $N$-small or $N$-large) experimental design was used, as we describe below. Specifically, for P-cycle trials, the sequence of displays was generated according to the $p$ value and the displays alternated between lower values of $p(<0.5)$ and higher values of $p(>0.5)$, with every two displays forming a cycle. As the consequence, the values of $p$ in $P$-cycle trials varied at a rhythm of $3.33 \mathrm{~Hz}$, while the values of $p(1-p)$ would be aperiodic. In contrast, for $U$-cycle trials ( $U$ for uncertainty), the sequence of displays was generated according to the value of $p(1-p)$ in a similar way so that the displays alternated between lower values of $p(1-p)(<0.21)$ and higher values of $p(1-p)(>0.21)$ in cycles of $3.33 \mathrm{~Hz}$, while the values of $p$ were aperiodic. In other words, in $P$-cycle trials, the $p$ value underwent a rhythmic fluctuation and the $p(1-$ p) value followed an aperiodic random course, whereas the opposite pattern occurred for $U$-cycle trials. Fig 1B illustrates the $P$-cycle (left) and $U$-cycle (right) conditions. Note that the discrete colors used in Fig $1 \mathrm{~B}$ to code the values of $p$ and $p(1-p)$ are only for visualization purposes. The actual values of $p$ and $p(1-p)$ were randomly chosen from continuous uniform distributions (see Methods). 
135 Our experimental design had two important features. First, the values of $p$ and

$136 p(1-p)$ were statistically independent of each other. Second, the values of $p$ and $p(1-$

$137 \quad p)$ were dissociated in periodicity. Note that subjects' task was always to attend to $p$

138 (relative frequency), and $p(1-p)$ was completely task-irrelevant in both $P$-cycle and $U$ -

139 cycle trials.

140 Besides, the total number of dots in a display (numerosity, denoted $N$ ) was not

141 constant but varied from display to display, independently of $p$ or $p(1-p)$, ranging from

14210 to 90 ( $N$-small trials) or from 100 to 900 ( $N$-large trials). The introduction of $N$-small

143 versus $\mathrm{N}$-large trials served as a means to manipulate the slope of probability distortion

144 [1] so as to reveal the possible links between behavior and brain activity.

146 Behavioral probability distortions quantified by LLO

147 All the 22 subjects performed well on reporting the relative-frequency of the last display,

148 whose subjective estimate $\pi(p)$ was highly correlated with the objective relative-

149 frequency $p$ (all Pearson's $r>0.71, P<0.001$ ). Given that the stimulus sequence might

150 end unexpectedly, if subjects had failed to track the sequence, they might have missed

151 the last stimulus. Subjects' sensible reports thus provide evidence that they had

152 tracked the change of $p$ throughout the trial as instructed.

153 Fig 2 A shows the reported $\pi(p)$ of one representative subject, who showed a

154 typical inverted-S-shaped probability distortion-overestimating small $p$ and

155 underestimating large $p$. To better visualize the deviation of $\pi(p)$ from $p$, we plot 
158 subjects exhibited the opposite S-shaped distortion, a profile also consistent with

159 previous findings [1, 4].

A

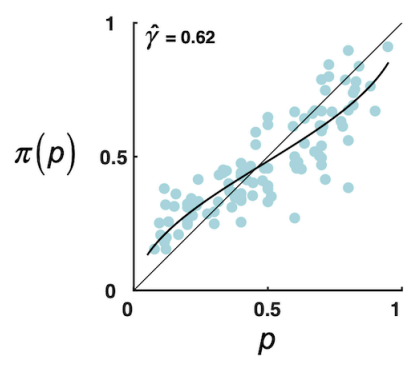

C

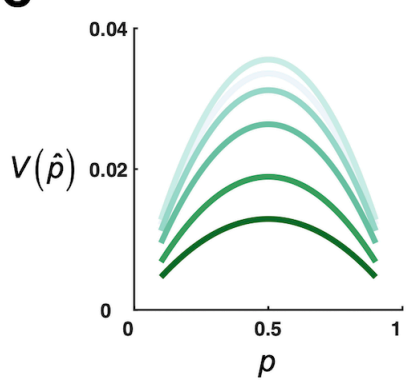

$\mathbf{F}$

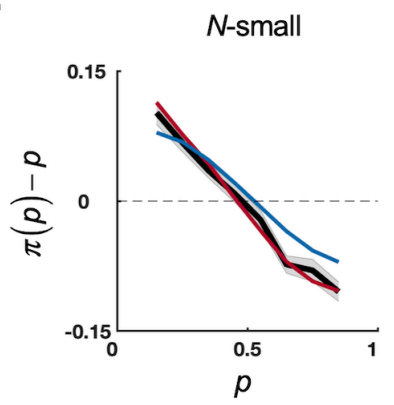

H

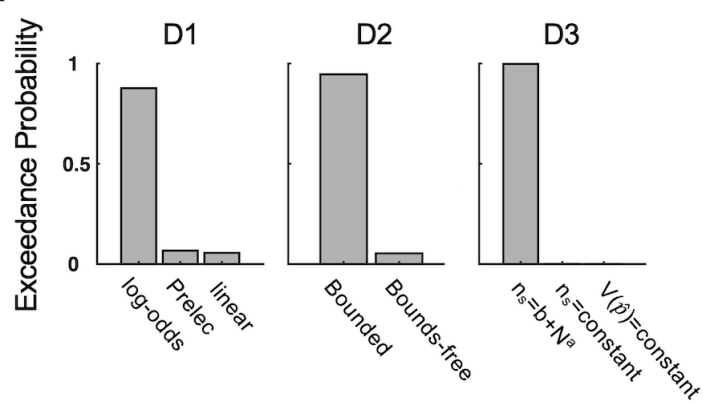

D

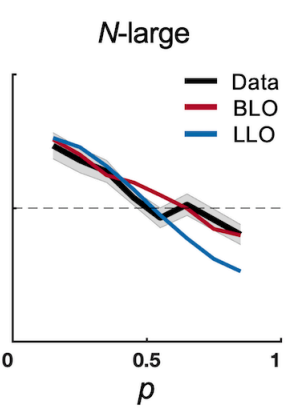

B

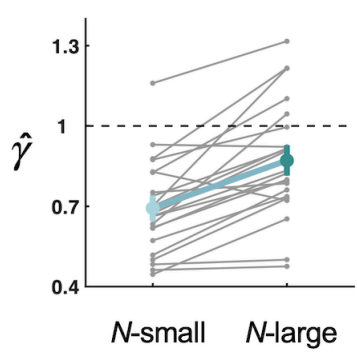

E
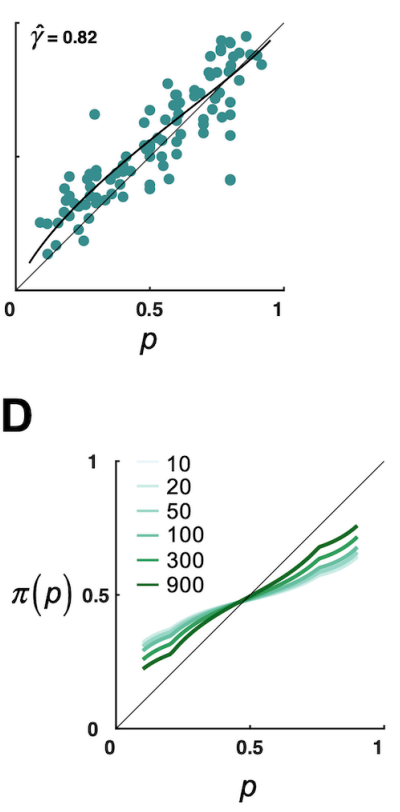

G

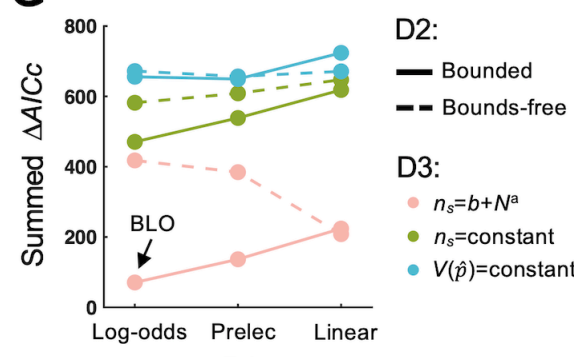

D1

I

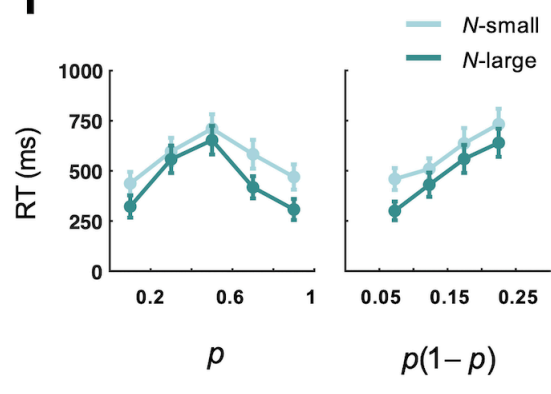

Fig 2. Behavioral and modeling results. 
164

165

166

167

168

169

170

171

172

173

174

175

176

177

178

179

180

181

182

183

184

185

186

187

188

189

190

191

192

194

separately for the $\mathrm{N}$-small and $\mathrm{N}$-large conditions. Each dot is for one trial. Black curves denote LLO model fits. (B) Slope of distortion $\hat{\gamma}$ for the $\mathbf{N}$-small and $\mathbf{N}$-large conditions. Each gray line is for one subject. The thick green line denotes the group mean. Error bars denote SEM. (C) Illustration of representational uncertainty $(\boldsymbol{V}(\hat{\boldsymbol{p}}))$ as a function of $\boldsymbol{p}$ and $\boldsymbol{N}$. Larger values of $N$ are coded in darker colors (same as $\mathbf{D}$ ). The maximal sample size is assumed to follow the form $n_{s}=b+N^{a}$, with $a=0.42$ and $b=1.91$ (median estimates across subjects). (D) Illustration of probability distortion predicted by BLO: $\boldsymbol{\pi}(\boldsymbol{p})$ as a function of $\boldsymbol{p}$ and $\boldsymbol{N}$. Larger values of $N$ are coded in darker colors. (E) Slopes of distortion: data versus model fits. According to the value of $N$ in the last display, all trials were divided into four bins and the slope of distortion, $\hat{\gamma}$, was estimated separately for each bin. Gray filled circles denote data. Error bars denote SEM. Red and blue circles respectively denote BLO and LLO model fits. $(F) \pi(p)-p$ as a function of $\boldsymbol{p}$ and $\boldsymbol{N}$. Black curves denote smoothed data on the group level, separately for the $N$ small and $N$-large conditions. Shadings denote SEM. Red and blue curves respectively denote BLO and LLO model fits. (G) Results of factorial model comparison: summed $\triangle$ AICc. Lower values of $\triangle \mathrm{AICc}$ indicate better fits. The BLO model outperformed all the alternative models. $(\mathrm{H})$ Protected exceedance probability on each model dimension. Each panel is for model comparison on one dimension. Each assumption of the BLO model outperformed the alternative assumptions on its dimension. (I) Response time as an additional index for representational uncertainty. The mean response time (RT) for subjects to initiate their relative-frequency report is plotted against binned $p$ or $p(1-p)$, separately for the $\mathrm{N}$-small and $\mathrm{N}$-large conditions. Error bars denote SEM.

We next used the LLO model to quantify such inverted-S- or S-shaped distortions, summarizing each subject's probability distortion behavior for each trial type $(P$-cycle or $U$-cycle) and dot numerosity ( $N$-small or $N$-large) conditions by two parameters fitted from $\pi(p)$ (see Methods): slope $\hat{\gamma}$ and crossover point $\hat{p}_{0}$. According to $2(P$-cycle or $U$-cycle) by 2 ( $N$-small or $N$-large) repeated-measures ANOVAs separately performed for the estimated $\hat{\gamma}$ and $\hat{p}_{0}$, numerosity showed a significant influence on $\hat{\gamma}\left(F(1,63)=57.82, P<0.001, \quad \eta_{p}^{2}=0.48\right)$ and a statistically significant but small influence on $\hat{p}_{0}\left(F(1,63)=4.24, P=0.044, \eta_{p}^{2}=0.06\right)$. No other main effects or 
195 interactions reached significance (all $P>0.26$ ). In particular, the $\hat{\gamma}$ estimated from $N$ -

196 large trials was greater than that of $\mathrm{N}$-small trials by $25.75 \%$ (Fig 2B). Thus our

197 subsequent analysis would mainly focus on $\hat{\gamma}$ and collapse the two cycle conditions

198 to estimate $\hat{\gamma}$. As illustrated in Fig 2A, a greater $\hat{\gamma}$ implies a less curved inverted-S-

199 shaped distortion (for $\hat{\gamma}<1$ ) or a more curved S-shaped distortion (for $\hat{\gamma}>1$ ).

200 It is noteworthy that LLO was only used as a way to quantify probability distortions

201 and their differences between conditions. The LLO model by itself could not explain

202 why the slope of distortion would differ between numerosity conditions, as observed

203 here (Fig 2AB). In contrast, the observed different $\hat{\gamma}$ between the $\mathrm{N}$-small and $\mathrm{N}$ -

204 large conditions could be well captured by the bounded log-odds (BLO) model [9],

205 which compensates for the $p(1-p)$ form of representational uncertainty, with greater

$206 \hat{\gamma}$ implying lower representational uncertainty. Next we will present the BLO model

207 and how it may account for our behavioral results.

\section{Bounded Log-Odds model and its behavioral evidence}

210 Zhang, Ren, and Maloney [9] proposed the BLO model as a rational account of

211 probability distortion, which can explain why probability distortion may vary with task

212 or individual. BLO has three main assumptions: (1) probability is internally represented

213 as log-odds, (2) representation is truncated to a bounded scale, and (3)

214 representational uncertainty is compensated in the final estimate of probability, so that

215 estimates associated with higher uncertainty will be discounted to a greater extent. 
216 See S1 Text for details. A full description of BLO would be out of the scope of the

217 present article and below we only focus on the uncertainty compensation assumption.

According to BLO, when representational uncertainty (denoted $V(\hat{p}))$ is higher,

219 the subjective estimate of probability is less contributed by the percept of the objective

220 probability and more by a prior estimate, which implies that the higher the

221 representational uncertainty, the shallower the slope of probability distortion. As

222 illustrated in Fig 2C, the representational uncertainty modeled in our experiment (see

223 S1 Text) is proportional to $p(1-p)$, that is, an inverted- $U$-shaped function of $p$.

224 Meanwhile, for a median subject, $V(\hat{p})$ first slightly increases with $N$ (Fig 2C, from

$225 N=10$ to $N=20)$ and then dramatically decreases with $N($ Fig $2 \mathrm{C}$, from $N=20$ to $N=900)$.

226 Consequently, BLO predicts that the slope of distortion $\hat{\gamma}$ is not necessarily constant,

227 but may slightly decrease with $N$ for very small $N$ and mostly increase with $N$ (Fig 2D),

228 which is indeed observed in our experiment (Fig 2B \& 2E).

We are aware that representational uncertainty $V(\hat{p})$ here is not equivalent but 230 proportional to $p(1-p)$. In the neural analysis we will present, we chose to focus on the

231 encoding of $p(1-p)$ (as a proxy for representational uncertainty) instead of $V(\hat{p})$,

232 because $V(\hat{p})$ was highly correlated with numerosity $N$ and thus would be difficult to

233 be separated from the latter in brain activities. In contrast, $p(1-p)$ and $N$ were

234 independent of each other by design. Besides, $p(1-p)$ has a nice connection with the

235 "second-order valuation" proposed by Lebreton et al. [18].

236 Model versus data. Zhang et al. [9] has provided behavioral evidence for the BLO

237 model in two different tasks including relative-frequency judgment. Here we performed 
238 similar tests on our behavioral data and fit the BLO model to the reported $\pi(p)$ for

239 each subject using maximum likelihood estimates. The performance of the LLO model

240 served as a baseline. When evaluating LLO's performance in fitting the data, we

241 estimated one set of parameters for all conditions instead of estimating different

242 parameters for different conditions as when we applied LLO as a measuring tool for

243 probability distortions. In Fig $2 \mathrm{~F}$, we plot the observed $\pi(p)-p$ (smoothed and

244 averaged across subjects) as a function of $p$ separately for different numerosity

245 conditions and contrasted it with the BLO and LLO model predictions. The BLO

246 prediction agreed well with the observed probability distortion function, while LLO

247 largely failed. The advantage of BLO over LLO was even more pronounced in plots for

248 individual subjects (S1 Fig).

249 We further examined how well BLO could predict the observed numerosity effect

250 in the slope of distortion. For each subject, we divided the trials of each numerosity

251 condition evenly into two bins according to the value of $N$ in the last display (i.e., the

252 display that was reported) and then estimated $\hat{\gamma}$ for each of the four bins. The pattern

253 of $\hat{\gamma}$ in real data-initial slight decrease and subsequent dramatic increase with $\mathrm{N}-$

254 was quantitatively predicted by the fitted BLO model (Fig 2E).

255 Factorial model comparison. Similar to Zhang et al. [9], we performed a factorial

256 model comparison [19] to test whether each of the three assumptions in the BLO model

257 outperformed plausible alternative assumptions in fitting behavioral data. Accordingly

258 we constructed $3 \times 3 \times 2=18$ models and fit each model for each subject using

259 maximum likelihood estimates (see S2 Text for details). The Akaike information 
260 criterion with a correction for sample sizes, AICc [20, 21], was used as the metric of

261 goodness-of-fit. Lower AICc indicates better fit. For each subject, the model with lowest

262 AICc was used as a reference to compute $\triangle \mathrm{AICc}$ for each model. According to the

263 summed $\triangle \mathrm{AICc}$ across subjects, BLO was the best model among the 18 models (Fig

$2642 G)$.

We also evaluated whether each of BLO's three assumptions was the best among

266 its alternatives on the same dimension. In particular, we used the group-level Bayesian

267 model selection [22-24] to compute the probability that each specific model

268 outperforms the other models in the model set ("protected exceedance probability")

269 and marginalized the protected exceedance probabilities for each dimension (i.e.,

270 adding up the protected exceedance probabilities across the other two dimensions).

271 Indeed, all three assumptions of the BLO model in the present study-log-odd,

272 bounded, and compensation for $V(\hat{p})$ (with $n_{s}=b+N^{a}$ )-outperformed their

273 alternatives with probabilities of $87.7 \%, 94.6 \%$ and $99.8 \%$, respectively (Fig $2 \mathrm{H}$ ).

274 Furthermore, we performed a model recovery analysis (similar to [25]) to confirm

275 that the advantage of BLO in factorial model comparison is real and does not result

276 from model mis-identification. In particular, we generated 50 synthetic datasets for

277 each of the 18 models. All the datasets generated from BLO were best fit by BLO. Out

278 of the 850 datasets generated from the other models, only $0.24 \%$ were mis-identified

279 to BLO (see S2A Fig for details).

280 We also verified that the parameters of BLO could be reasonably well identified

281 (S2B Fig). Even if some of the BLO parameters were not perfectly identified, it would 
not influence the results of the neural analysis we report below, most of which did not rely on the estimated BLO parameters.

\section{Evidence for representational uncertainty in response time}

285 The above modeling analysis on subjects' reported relative frequency (i.e. model fits

286 as well as the factorial model comparison) suggests that subjects might compensate

287 for representational uncertainty that is proportional to $p(1-p)$ and the $N$-large condition 288 was associated with lower uncertainty than the $N$-small condition.

The response time (RT) of reporting relative frequency (defined as the interval

290 between the response screen onset and the first mouse move) provides an additional

291 index for representational uncertainty, given that lower uncertainty would lead to 292 shorter RTs. We divided the values of $p$ into five bins and computed RT for each bin 293 and separately for the $N$-small and $N$-large trials. According to a 5 ( $p$ bins) by $2(N-$ 294 small vs. $N$-large) repeated-measures ANOVA on RTs, both the main effects of the $p$ 295 value $\left(F(4,189)=29.02, P<0.001, \quad \eta_{p}^{2}=0.38\right)$ and numerosity $(F(1,189)=25.61, P$ $\left.296<0.001, \eta_{p}^{2}=0.12\right)$ were significant. Consistent with Lebreton et al. [18], RTs were 297 longest at $p=0.5$ and shortest when $p$ was close to 0 or 1 (Fig 2I, left). More precisely, 298 RTs increased almost linearly with $p(1-p)$ (Fig 2I, right), in agreement with what one 299 would expect if representational uncertainty is proportional to $p(1-p)$. Besides, RTs 300 were shorter in $N$-large trials than in $N$-small trials, which echoes the lower $V(\hat{p})$ for 301 larger $N$ (Fig 2C) and provides further evidence that the $N$-large condition was 302 accompanied by lower representational uncertainty. 
In sum, we found that a considerable portion of variability in subjects' probability

distortion functions and RT patterns can be accounted by differences in

305 representational uncertainty. One may wonder whether the effects of representational

306 uncertainty can be explained away by task difficulty. We doubt not, because higher

307 representational uncertainty does not necessarily correspond to higher task difficulty.

308 For example, representational uncertainty for relative-frequency estimation is

309 proportional to $p(1-p)$, which is maximal at $p=0.5$ and minimal when $p$ is close to 0 or

310 1. In contrast, regarding difficulty, there seems to be little reason to expect that relative-

311 frequency estimation should be more difficult for $p=1 / 2$ than for $p=1 / 3$, or be more

312 difficult for $p=1 / 3$ than for $p=1 / 4$. As another counter-example, representational

313 uncertainty modeled in the present study is higher in the $\mathrm{N}$-small condition than in the

$314 N$-large condition (Fig 2C), but there seems to be little reason to expect relative-

315 frequency estimation to be more difficult for displays with fewer dots.

317 Neural entrainment to periodic changes of $p$ or $p(1-p)$

318 After showing that the BLO models can well capture the behavioral results, we next

319 examined whether the brain response could track the periodically changing $p$ values

320 ( $P$-cycle) or $p(1-p)$ values $(U$-cycle) in the stimulus sequence. Recall that the $P$-cycle

321 and U-cycle trials had identical individual displays and differed only in the ordering of

322 the displays: In $P$-cycle trials, $p$ alternated between small and large values in cycles of 
$323 \quad 3.33 \mathrm{~Hz}$ while $p(1-p)$ was aperiodic; in $U$-cycle trials, $p(1-p)$ alternated in cycles of 3.33

$324 \quad \mathrm{~Hz}$ while $p$ was aperiodic.

As shown in Fig 3, the brain response indeed tracked the periodic changes of $p$

326 and $p(1-p)$. In particular, in $P$-cycle trials (Fig 3, left), the phase coherence between

327 the periodic $p$ values and the MEG time series reached significance at $3.33 \mathrm{~Hz}$

328 (permutation test, $F D R$ corrected $P_{F D R}<0.05$ ), mainly in the occipital and parietal

329 sensors. Importantly, significant phase coherence was also found at $3.33 \mathrm{~Hz}$ in $U$-cycle

330 trials between the periodic $p(1-p)$ values and the MEG time series (Fig 3, right). That

331 is, the brain activity was also entrained to the periodic changes of the representational

332 uncertainty of $p$ (i.e. $p(1-p)$ ). Given that subjects were only asked to track the value of

$333 p$ but not $p(1-p)$, the observed neural entrainment to the task-irrelevant $p(1-p)$

334 suggests an automatic encoding of representational uncertainty in the brain.

$\boldsymbol{P}$-cycle $\quad \boldsymbol{U}$-cycle
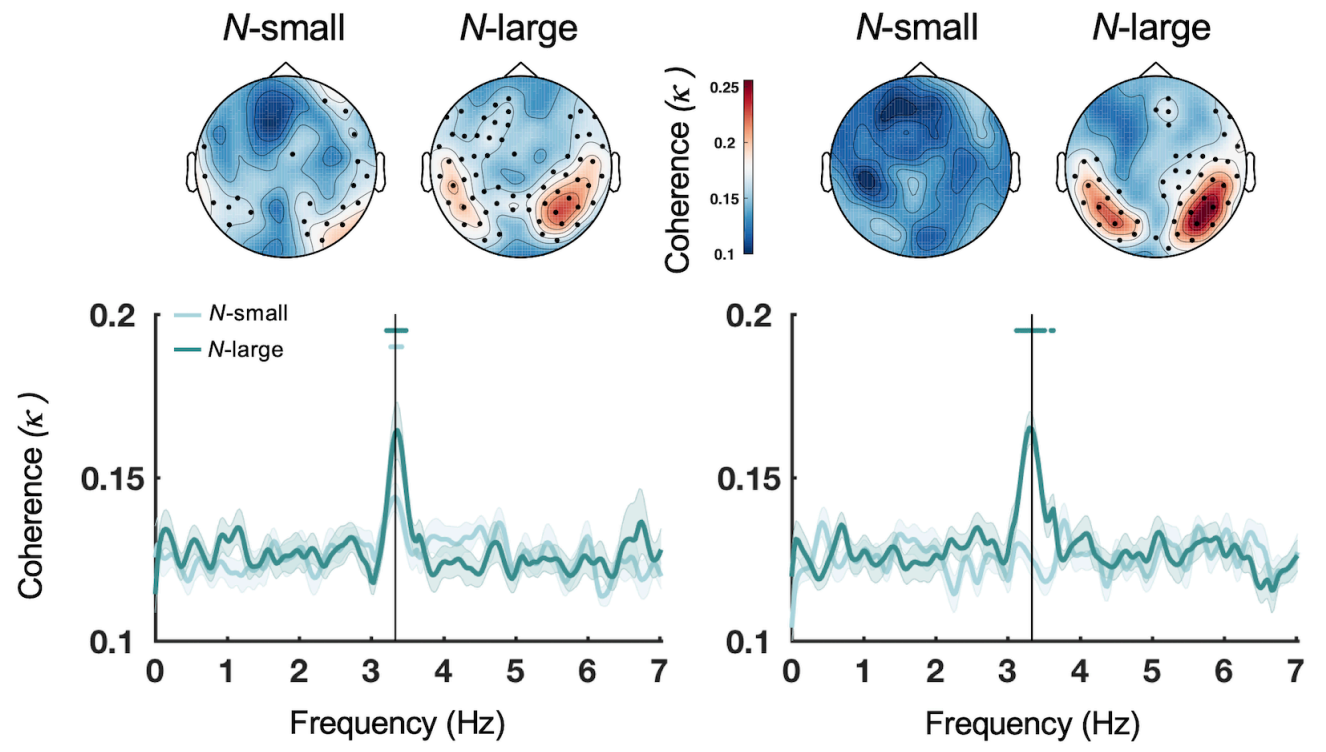

Fig 3. Results of phase coherence analysis.

337 Grand-averaged phase coherence spectrum for magnetometers. Left: The phase 338 coherence $(\kappa)$ between the periodic $p$ values and MEG time series in $P$-cycle trials. Right: 339 The phase coherence between the periodic $p(1-p)$ values and MEG time series in $U$-cycle 

trials. Light and dark green curves respectively denote the $\mathrm{N}$-small and $\mathrm{N}$-large conditions. Shadings denote SEM across subjects. The vertical line marks $3.33 \mathrm{~Hz}$. Dots above the spectrum mark the frequency bins whose phase coherence was significantly above chance level (permutation tests, $P_{F D R}<0.05$ with FDR correction across frequency bins). Insets: Grand-averaged phase coherence topography at $3.33 \mathrm{~Hz}$ for each cycle and numerosity condition. Solid black dots denote sensors whose phase coherence at $3.33 \mathrm{~Hz}$ was significantly above chance level (permutation tests, $P_{F D R}<0.05$ with FDR corrected across magnetometers).

Notably, the significant phase coherences between the periodic $p$ or $p(1-p)$ values and MEG time series were not simply because the MEG time series consisted of 3.33 $\mathrm{Hz}$ frequency components. In a control analysis, we calculated the phase coherence between the aperiodic variable (i.e. $p$ in $U$-cycle trials or $p(1-p)$ in $P$-cycle trials) and the same MEG time series, and found that there were no significant peaks at $3.33 \mathrm{~Hz}$ (S5B Fig). Moreover, we had carefully controlled potential confounding variables in our experimental design. For example, $p$ and $p(1-p)$ were linearly independent of each other, both of which had negligible correlations with numerosity or low-level visual features such as luminance, contrast, and color variance (S5A Fig). Furthermore, different from $p$ and $p(1-p)$, these potential confounding variables were associated with similar entrainment responses in $P$-cycles and $U$-cycles (S5B Fig).

\section{Behavioral probability distortion and the neural entrainment to $p$ and $p(1-$}

\section{p)}

We next examined the relationship between behavioral probability distortion and the neural entrainment to $p$ and $p(1-p)$ on a subject-by-subject basis. Specifically, the phase coherence between a specific variable $(p$ or $p(1-p))$ and the MEG time series 
366 can be considered as a measure of the strength of neural responses to the variable

367 [26]. We defined

368

$$
\beta=\frac{\kappa_{p}}{\kappa_{p(1-p)}}
$$

369 to quantify the relative strength of $p$ to $p(1-p)$ in neural responses, where $\kappa_{p}$ denotes

370 the phase coherence for $p$ in $P$-cycle trials, and $\kappa_{p(1-p)}$ denotes the phase coherence

371 for $p(1-p)$ in $U$-cycle trials. A higher value of $\beta$ would imply a stronger neural

372 encoding of relative-frequency or a weaker encoding of representational uncertainty

373 and is thus supposed to yield probability distortions of a greater slope [9]. Note that

374 both the behavioral and neural measures we defined below were intra-subject ratios

375 that were unitless and scale free, thus not subject to the potential scaling issues in 376 inter-individual correlation [27].

377 Given that the estimated slope of distortion $\hat{\gamma}$ was greater in $N$-large trials than

378 in $N$-small trials, we would expect $\beta$ to change in the same direction across the

379 numerosity conditions. That is, suppose we define $\Delta \hat{\gamma}=\ln \left(\frac{\hat{\gamma}_{N \text {-large }}}{\hat{\gamma}_{N \text {-small }}}\right)$ and

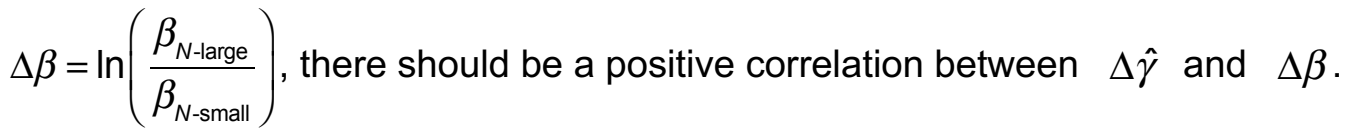

As shown in Fig 4, there was indeed a significant correlation between behavioral

382 and neural measures across subjects in the parietal region (Pearson's $r=0.67, F D R$ -

384 with probability distortion behaviors. 


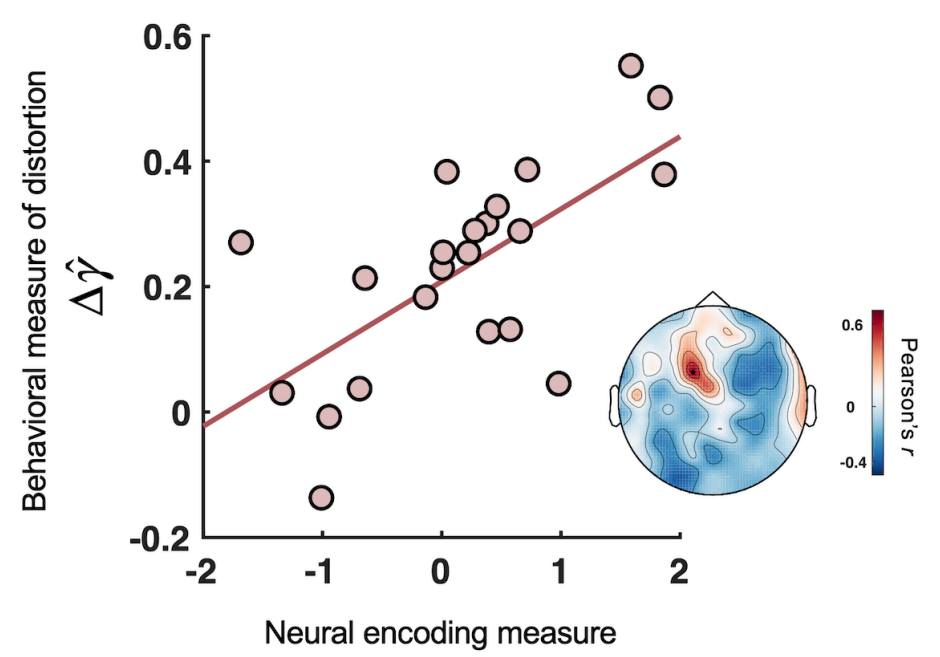

$\Delta \beta$

Fig 4. Neural responses to $p$ and $p(1-p)$ predict the slope of probability distortion. We defined $\Delta \hat{\gamma}=\ln \left(\frac{\hat{\gamma}_{N \text {-arge }}}{\hat{\gamma}_{N \text {-smal }}}\right)$ and $\Delta \beta=\ln \left(\frac{\beta_{N \text {-arge }}}{\beta_{N \text {-smal }}}\right)$ to respectively quantify how much the behavioral measure of the slope of distortion, $\hat{\gamma}$, and the relative strength of $p$ to $p(1-p)$ in neural responses, $\beta$, changed across the two numerosity conditions. Main plot: Correlation between $\Delta \hat{\gamma}$ and the $\Delta \beta$ at the parietal magnetometer channel MEG0421. The logarithm transformation was used only for visualization. Each dot denotes one subject. Inset: Correlation coefficient topography between $\Delta \hat{\gamma}$ and $\Delta \beta$, on which MEG0421 is marked by a solid black dot.

\section{Neural encoding of $p$ and $p(1-p)$ over time and the associated brain} regions

After establishing the automatic tracking of cyclic changes in $p(1-p)$ and its behavioral relevance, we next aimed to delineate the temporal dynamics of $p$ and $p(1-p)$ in the brain signals. Recall that on each trial one of the two variables of our interest $(p$ or $p(1-$ p)) changed periodically and the other was aperiodic. Therefore, we could decode the temporal course of aperiodic $p(1-p)$ in $P$-cycles trials and aperiodic $p$ in $U$-cycle trials. In particular, we performed a time-resolved decoding analysis based on all 306 sensors (see Methods) using a regression approach that has been employed in 
404 previous EEG and MEG studies [28, 29] including ours [30-32]. The intuition of the

405 time-resolved decoding analysis is as follows. Suppose the onset of each new $p$ or

$406 p(1-p)$ value in the stimulus sequence evokes phase-locked brain responses that

407 extend across time and whose magnitudes are proportional to the encoded value. The

408 resulting MEG time series would then be a superposition of the responses to all the

409 preceding stimuli in the sequence. But as soon as the values of an encoded variable

410 are not correlated over time (i.e. free of autocorrelation), their brain response profiles

411 are separable. In particular, for any stimulus in the sequence, we can use the MEG

412 signals at a specific delay after the stimulus onset to predict the value of the stimulus.

413 Importantly, different from the phase coherence analysis (Fig 3) that only reflects the

414 overall strength of the brain responses, this time-resolved decoding analysis allows us

415 to assess how $p$ and $p(1-p)$ are encoded over time.

$416 \quad$ Fig 5 plots the temporal course of the decoding performance for $p$ (left) and $p(1-$

$417 p)$ (right). We found that both $p$ and $p(1-p)$ were successfully decoded from the MEG

418 signals in $N$-large trials (cluster-based permutation test, $\left.P_{\text {cluster }}<0.05\right)$. In particular, the

419 decoding performance for $p$ peaked around $327 \mathrm{~ms}$ after stimulus onset (Fig 5, left),

420 while that for $p(1-p)$ peaked around 419 ms (Fig 5, right). In other words, the neural

421 encodings of relative frequency and its representational uncertainty had distinct time

422 courses, with the latter occurring approximately $100 \mathrm{~ms}$ later than the former (also see

423 S8 Fig for the decoding performance of individual subjects).

424 In contrast, none of the potential confounding variables (numerosity, luminance,

425 etc.) showed temporal courses similar to $p$ or $p(1-p)$ when the same time-resolved 
426 decoding procedure was applied (S5C Fig). The automatic encoding of the task-

427 relevant $p(1-p)$ might seem surprising. To further verify that this was not an artifact of

428 potential low-level confounds, we performed an additional decoding analysis for $p(1-$

$429 p$ ) based on the cross-validated version of confound regression (CVCR) [33], where

430 the confounding variables were regressed out from MEG time series before time-

431 resolved decoding analysis (see S7 Text for details). The decoded temporal course for

$432 p(1-p)$ was little changed (S9A Fig). A similar decoding analysis for the complete form

433 of representational uncertainty $V(\hat{p})$ resulted in similar temporal course as that of

$434 p(1-p)$ (S9B Fig). In sum, we found automatic encoding of $p(1-p)$ in brain signals even

435 when it was task-irrelevant and when low-level confounding factors were excluded.

436 Further, based on the time windows that achieved the highest time-resolved

437 decoding performance, we performed a similar decoding analysis at each sensor

438 location (including one magnetometer and two orthogonal planar gradiometers)

439 separately for $p$ and $p(1-p)$ to assess the associated brain regions (see Methods). We

440 found that the encoding of the two variables involved overlapping parietal-occipital

441 regions (Fig 5, see S7 Fig for the results of the other above-chance time windows),

442 including the parietal region where $\Delta \beta$ and $\Delta \hat{\gamma}$ were positively correlated (Fig 4). 


\section{Decoding $\boldsymbol{p}$}

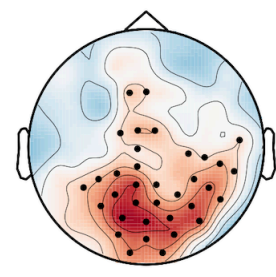

$225-350 \mathrm{~ms}$

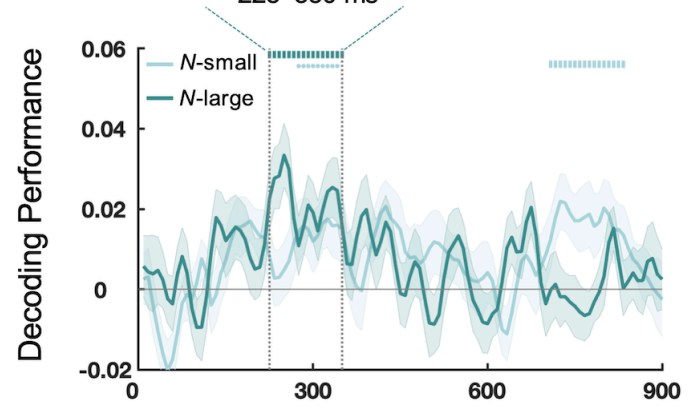

Time after stimulus onset (ms)
Decoding $\boldsymbol{p}(\mathbf{1}-\boldsymbol{p})$

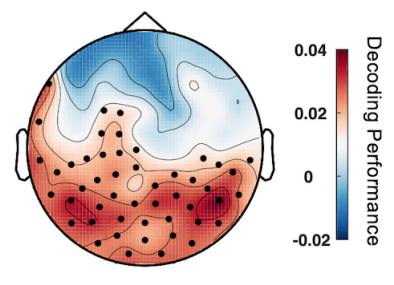

$308-475 \mathrm{~ms}$

Fig 5. Results of decoding analyses.

Main plots: Time-resolved decoding performance over different time lags for $p$ (left panel) and $p(1-p)$ (right panel), separately for the $\mathrm{N}$-small (light green) and $\mathrm{N}$-large (dark green) conditions. Shadings denote SEM across subjects. Symbols above the curves indicate time lags that had above-chance decoding performance (cluster-based permutation tests), with vertical bars representing $P_{\text {cluster }}<0.01$ and dots representing $0.01 \leq P_{\text {cluster }}<0.05$. The decoding performance for $p$ peaked at around $327 \mathrm{~ms}$ after stimulus onset, while that for $p(1-p)$ peaked at around $419 \mathrm{~ms}$. Insets: Topography of spatial decoding performance for the time window (highlighted by the funnel-shaped dashed lines) that contained the peak decoding performance in the time-resolved decoding analysis. Solid black dots indicate the sensor locations with above-chance decoding performance $\left(P_{F D R}<0.05\right)$ for the $N$ large condition. (Spatial decoding in the same time windows for the $\mathrm{N}$-small condition resulted in no statistically significant sensors.) The encodings of $p$ and $p(1-p)$ involved overlapping parietal-occipital regions, including the parietal region where $\Delta \beta$ and $\Delta \hat{\gamma}$ were positively correlated (Fig 4). 


\section{Discussion}

462 We used MEG to investigate probability distortions in a relative-frequency estimation

463 task and found that the human brain encodes not only the task-relevant relative

464 frequency but also its task-irrelevant representational uncertainty. The neural encoding

465 of the representational uncertainty occurs at as early as approximately $400 \mathrm{~ms}$. These

466 results suggest that the human brain automatically and quickly quantifies the

467 uncertainty inherent in probability information. Our findings provide neural evidence for

468 the theoretical hypothesis that probability distortion is related to representational

469 uncertainty. More generally, these findings may connect to the functional role of 470 confidence (estimation of uncertainty) during judgment and decision making.

\section{Neural computations underlying probability distortions}

473 Humans show highly similar probability distortions in tasks involving probability or

474 relative frequency, with the subjective probability typically an inverted-S-shaped

475 function of the objective probability [1]. Why do people distort probability information in such a systematic way? Though inverted-S-shaped distortions of probability had been

477 found in the activity of a few brain regions [34, 35], the neural computations involved

478 in transforming objective probabilities to subjective probabilities were largely unknown.

479 Several theories $[9,15-17]$ rationalize probability distortion as a consequence of 480 compensating for representational uncertainty, in accordance with the framework of 481 Bayesian Decision Theory [36]. In brief, the brain will discount an internal 

human representation of probability or relative frequency. uncertainty $p(1-p)$ is not just virtual quantities assumed in computational models of probability distortions but is really computed in the brain.

\section{Representational uncertainty and confidence}

495 The representational uncertainty that concerns us lies in the internal representation of probability or relative-frequency. One concept that may occasionally coincide with such

497 epistemological uncertainty but is entirely different is outcome uncertainty, which is 498 widely studied in the literature of decision under risk [37, 38], prediction error [37] and 499 surprise $[39,40]$. For example, for a two-outcome gamble with probability $p$ to receive $500 x_{1}$ and probability $1-p$ to receive $x_{2}$, the variance of the outcomes is proportional to $501 p(1-p)$. In the present study, the relative-frequency estimation task we chose to 502 investigate does not involve similar binary uncertain outcomes and thus avoids the 
503 possible confusion of outcome uncertainty with representational uncertainty.

504 Therefore, the neural encoding of $p(1-p)$ we found here cannot be attributed to the

505 perception of outcome uncertainty.

In fact, representational uncertainty is more closely related to confidence, except

507 that it is not necessarily bound to any specific judgment or decision [41]. Specifically,

508 the encoding of representational uncertainty may be understood as a special case of

509 the "second-order valuation" proposed by Lebreton et al. [18]. As described earlier,

510 Lebreton et al. [18] found a principled relationship between an overt numerical

511 judgment and the individual's confidence about the judgment, with the latter being a

512 quadratic function of the former. They showed in fMRI studies that such quadratic

513 form - as a proxy to confidence-is automatically encoded in vmPFC, even when

514 confidence rating is not explicitly required of. Using the same quadratic form as the

515 proxy, here we found automatic encoding of the representational uncertainty of relative

516 frequency during the tracking of a visual sequence. Meanwhile, our findings extend

517 previous work and contribute to the confidence literature in the following three aspects.

518 First, we found automatic encoding of representational uncertainty even in the

519 absence of overt judgment, not just in the absence of overt confidence estimation. In

520 our experiment, subjects were asked to track the relative frequency in the stimulus

521 sequence but made no overt judgment except for the last display. Moreover, displays

522 were refreshed every $150 \mathrm{~ms}$, apparently leaving no time for deliberation.

523 Second, we resolved the temporal course of the encoding of representational

524 uncertainty, which would be inaccessible under the low temporal resolution of fMRI. In 
525 particular, representational uncertainty is encoded as early as 400 ms upon stimulus

526 onset, approximately $100 \mathrm{~ms}$ after the encoding of relative frequency itself. The

527 occipital-parietal region is involved in the processing of both relative-frequency and its

528 representational uncertainty but during different time windows. The fast encoding of

529 representational uncertainty we found echoes recent psychophysiological findings in

530 humans [42-44] and non-human primates [45] that the confidence encoding for

531 perceptual judgments can be detected in the brain far before confidence rating and

532 even prior to the overt judgment or decision making.

533 Third, our findings connect to the functional importance of confidence encoding in

534 information processing $[41,46,47]$ that is still not well understood but receives growing

535 attention. In particular, here we ask how the neural encoding of representational

536 uncertainty may relate to probability distortion. By comparing experimental conditions

537 under which the same individuals' distortions of relative frequency differed, we found

538 that a relatively stronger response to representational uncertainty in the parietal region

539 corresponds to a shallower slope of distortion. We conjecture that the automatic, fast

540 encoding of representational uncertainty might not just be post hoc evaluation but

541 indeed regulate probability distortion. Meanwhile, we are aware that our current

542 findings are based on correlational analyses and whether there is causality between

543 the neural encoding of representational uncertainty and probability distortion still

544 awaits future empirical tests. 


\section{Methodology implications}

547 The "steady-state responses" (SSR) technique [48]—using rapid periodic stimulus

548 sequence to entrain brain responses-has been widely used with EEG/MEG to

549 investigate low-level perceptual processes, which increases the signal-to-noise ratio

550 of detecting the automatic brain responses to the stimuli by sacrificing temporal

551 information. In our experiment, we constructed periodic $p$ or $p(1-p)$ sequences and

552 showed that SSR can also be used to reveal the brain's automatic responses to these

553 more abstract variables. Moreover, we demonstrate the feasibility to perform time-

554 resolved decoding for the other aperiodic variable embedded in the same sequence,

555 whereby exploiting the advantages of both the SSR and time-resolved decoding

556 techniques. Such design would be useful for a broad range of problems that need to

557 dissociate the processing of two or more variables in brain activities.

558

559 


\section{Materials and Methods}

\section{Ethics Statement}

562 The study had been approved by the Institutional Review Board of School of

563 Psychological and Cognitive Sciences at Peking University (\#2015-03-13c). Subjects

564 provided written informed consent in accordance with the Declaration of Helsinki.

565

566 Subjects

567 Twenty-two human subjects (aged 18-27, 13 female) participated. All of them had

568 normal or corrected-to-normal vision and passed the Farnsworth-Munsell 100 Hue

569 Color Vision Test [49].

\section{Experimental Procedures}

572 Apparatus. Subjects were seated approximately $86 \mathrm{~cm}$ in front of a projection screen

573 (Panasonic PT-DS12KE: $49.6 \times 37.2 \mathrm{~cm}, 1024 \times 768$ pixels, $60-\mathrm{Hz}$ refresh rate) inside

574 the magnetically shielded room. Stimuli were controlled by a Dell computer using

575 Matlab and PsychToolbox-3 [50, 51]. Subjects' behavioral responses were recorded

576 by a MEG-compatible mouse system (FOM-2B-10B from Nata technologies) and their

577 brain activities by a 306-channel MEG system (see MEG Acquisition and

578 Preprocessing for details).

579 Task. Each trial started with a white fixation cross on a blank screen for $600 \mathrm{~ms}$,

580 following which a sequence of displays of orange and blue dots was presented on a

581 gray background at a rate of $150 \mathrm{~ms}$ per display (Fig 1A). Subjects were asked to fixate 
582 on the central fixation cross and track the relative-frequency of each display. After the

583 sequence ended for $1000 \mathrm{~ms}$, a horizontal scale (0 to 100\%) appeared on the screen

584 and subjects were required to click on the scale to indicate the relative-frequency of

585 orange (or blue) dots on the last display. Half of the subjects reported relative frequency

586 for orange dots and half for blue dots.

587 To encourage subjects to pay attention to each display, one out of six trials were

588 catch trials whose duration followed a truncated exponential distribution (1-6 s, mean

$5893 \mathrm{~s}$ ), such that almost each display could be the last display. The duration of formal

590 trials was 6 or $6.15 \mathrm{~s}$. Only the formal trials were submitted to behavioral and MEG

591 analyses.

592 On each display, all dots were randomly scattered without overlapping within an

593 invisible circle that subtended a visual angle of $12^{\circ}$. The visual angle of each dot was

$5940.2^{\circ}$ and the center-to-center distance between any two dots was at least $0.12^{\circ}$. The

595 two colors of the dots were isoluminant $\left(\mathrm{ClE}_{\text {orange }}=[43,19.06,52.33], \mathrm{ClE}_{\text {blue }}=[43,-\right.$

$59615.49,-23.72])$. Isoluminant dark gray pixels $\left(\mathrm{ClE}_{\text {gray }}=[43,0,0]\right)$ were filled on the gray

597 background $\left(\mathrm{ClE}_{\text {background }}=[56.5,0,0]\right)$ between the dots as needed to guarantee each

598 display had equal overall luminance, which prevented luminance from being a

599 confounding factor for any abstract variables of interest.

600 Design. We adopted a steady-state response (SSR) design, which could achieve a

601 higher signal-to-noise ratio than the conventional event-related design [48]. The basic

602 idea was to vary the value of a variable periodically at a specific temporal frequency

603 and to observe the brain activities at the same frequency as an idiosyncratic response 
604 to the variable. The variables of most interest in the present study were relative

605 frequency $p$ and its representational uncertainty quantified by $p(1-p)$.

606 For half of the trials (referred as the $P$-cycle condition), the value of $p$ in the

607 sequence was chosen from uniform distributions with the ranges alternatively being

$608(0.1,0.5)$ and $(0.5,0.9)$ so that the sequence of $p$ formed cycles of $3.33 \mathrm{~Hz}$. For the 609 other half trials (referred as the $U$-cycle condition), the value of $p$ was chosen 610 alternatively from $(0.10 .3) \cup(0.7,0.9)$ and $(0.3,0.7)$ so that the sequence of $p(1-p)$

611 (i.e. proxy for uncertainty) was in cycles of $3.33 \mathrm{~Hz}$. That is, the two cycle conditions 612 had matched individual displays but differed in which variable formed a periodic 613 sequence. Accordingly, the $p(1-p)$ sequence in the $P$-cycle condition and the $p$ 614 sequence in the U-cycle condition were aperiodic, which had little autocorrelations (S3 615 Text \& S4 Fig).

616 The total number of dots on a display (numerosity, denoted $N$ ) was varied across

617 trials as well as across individual displays of the same trial. The value of $N$ for a specific

618 display was independent of the value of $p$ and generated as a linear transformation of

619 a beta random variable following $\operatorname{Beta}(0.1,10)$ so that the number of dots in each color $620(p N$ or $(1-p) N)$, a possible confounding variable of $p$, would only have moderate 621 correlations (Pearson's $|r|<0.5$ ) with $p$. The linear transformation was chosen to locate $622 N$ to the range of $[10,90]$ for half of the trials (the $N$-small condition) and to $[100,900]$ 623 for the other half (the $N$-large condition).

624 The numbers of dots in each color was $p N$ or $(1-p) N$ rounded to the nearest integer. 625 As the result of rounding, the actual relative-frequency was slightly different from the 
626 originally chosen $p$. In data analysis, we would use $p$ to denote the actual relative-

627 frequency that was presented to subjects.

628 To summarize, there were 2 ( $P$-cycle vs. $U$-cycle) by 2 ( $N$-small vs. $N$-large)

629 experimental conditions, with all conditions interleaved and each condition repeated

630 for 6 trials in each block. Each subject completed 10 blocks of 24 trials, resulting in 50

631 formal trials and 10 catch trials for each condition. Before the formal experiment, there

632 were 32 practice trials (the first 20 trials consisted of one display and the following 12

633 trials had the same settings as the formal experiment) for subjects to be familiar with

634 the procedure. No feedback was available during the experiment.

635

636 Behavioral Analysis

637 Measures of probability distortion. According to Zhang and Maloney [1], inverted-

638 S- or S-shaped probability distortions can be well captured by the LLO model

$$
\lambda[\pi(p)]=\gamma \lambda[p]+(1-\gamma) \lambda\left[p_{0}\right]+\varepsilon_{\lambda}
$$

640 where $p$ and $\pi(p)$ respectively denote the objective and subjective probability or

641 relative-frequency, $\lambda[p]=\log \frac{p}{1-p}$ is the log-odds transformation, and $\varepsilon_{\lambda}$ is

642 Gaussian error on the log-odds scale with mean 0 and variance $\sigma_{\lambda}^{2}$. The $\gamma, p_{0}$, and

$643 \sigma_{\lambda}$ are free parameters. The parameter $\gamma$ is the slope of distortion, with $\gamma<1$

644 corresponding to inverted-S-shaped distortion, $\gamma=1$ to no distortion, and $\gamma>1$ to $S$ -

645 shaped distortion. The parameter $p_{0}$ is the crossover point where $\pi(p)=p$.

646 For each subject and condition, we fit the reported relative-frequency to the LLO

647 model (Eq. 2) and used the estimated $\hat{\gamma}$ and $\hat{p}_{0}$ as the measures of relative- 
648 frequency distortions.

649 Response time. The response time (RT) was defined as the interval between the

650 response screen onset and subjects' first mouse move. The RT of the first trial of three

651 subjects was mis-recorded due to technical issues and was excluded from further

652 analysis. We divided all trials evenly into 5 bins based on the to-be-reported $p$, and

653 computed the mean $p$ and mean RT for each bin. A 5 ( $p$ bins) by 2 (numerosity

654 conditions) repeated-measures ANOVA was performed on the mean RTs. A similar

655 binning procedure was applied to $p(1-p)$ to visualize the relationship of RT to $p(1-p)$.

656 Non-parametric measures of probability distortion. As a complement to the LLO

657 model (Eq. 2), we used a non-parametric method to visualize the probability distortion

658 curve for each subject. In particular, we smoothed $\pi(p)-p$ using a kernel regression

659 method with the commonly-used Nadaraya-Watson kernel estimator [52-54]:

660

$$
\hat{M}_{h}(x)=\frac{\sum_{i=1}^{m} K\left(\frac{x-x_{i}}{h}\right) y_{i}}{\sum_{i=1}^{m} K\left(\frac{x-x_{i}}{h}\right)},
$$

661 where $x_{i}$ and $y_{i}(i=1,2, \ldots, m)$ denote observed pairs of stimuli and responses,

$662 \hat{M}_{h}(x)$ denotes the smoothed response at the stimulus value $x$, and $h$ is a parameter

663 that controls the degree of smoothing and were set to be 0.03 . The $K(\cdot)$ denotes the

664 Gaussian kernel function:

665

$$
K(z)=\frac{1}{\sqrt{2 \pi}} \exp \left(-\frac{z^{2}}{2}\right)
$$

667 Model fitting. For each subject, we fit the BLO model to the subject's $\pi(p)$ in each 
668 trial using maximum likelihood estimates. The fminsearchbnd (J. D’Errico), a function

669 based on fminsearch in MATLAB (MathWorks), was used to search for the parameters

670 that minimized negative log likelihood.

671 We compared the goodness-of-fit of the BLO model with all of the alternative

672 models based on the Akaike information criterion with a correction for sample sizes

673 (AICc) $[20,21]$ and group-level Bayesian model selection [22-24]. To verify that we had

674 found the global minimum, we repeated the searching process for 1000 times with

675 different starting points.

676

MEG Acquisition and Preprocessing

678 Subjects' brain activity was recorded by a 306-channel whole-head MEG system

679 (Elekta-Neuromag, 102 magnetometers and 102 pairs of orthogonal planar

680 gradiometers). Head position was measured before each block by an isotrack

681 polhemus system with four head position indicator coils (two on the left and right

682 mastoid, the other two on the left and right forehead below the hairline). Subjects

683 whose between-block head movement exceeded $3 \mathrm{~mm}$ would be excluded from further

684 analysis. Horizontal and vertical Electro-oculograms were recorded to monitor eye-

685 movement artifacts. Sampling rate was set to be $1000 \mathrm{~Hz}$ and an analog band-pass

686 filter from 0.1 to $330 \mathrm{~Hz}$ was applied. Maxwell filtering was used to minimize external

687 magnetic interference and to compensate for head movements [55, 56].

688 Standard preprocessing procedures were applied using Matlab 2016b and the

689 FieldTrip package [57]. The MEG data of each block was first low-pass filtered below 
$69020-\mathrm{Hz}$ and then segmented into epochs of $7.6 \mathrm{~s}$ relative to trial onset $(-0.6 \mathrm{~s}$ to $7 \mathrm{~s})$.

691 Independent component analysis (ICA) was applied to aggregated epoch data to

692 remove artifacts including blinks, eye movements, breaths and heart activity. No

693 subject was excluded for excessive head movement or other artifacts. For two subjects,

694 the first trial of one block was excluded due to the failure of synchronization between

695 stimulus onset and MEG recording onset at the beginning of the block.

\section{Phase Coherence Analysis}

698 Given a periodically-changed stimulus sequence ( $p$ in $P$-cycle or $p(1-p)$ in $U$-cycle),

699 stimulus-evoked brain responses imply that the phase difference between the stimulus

700 and response time series should be coherent across trials at the stimulus frequency

701 [58]. The phase coherence across trials is defined as [26]:

702

$$
\kappa=\frac{\left|\bar{P}_{r s}\right|}{\sqrt{\bar{P}_{s s}} \sqrt{\bar{P}_{r r}}},
$$

703 where $\bar{P}_{S S}$ and $\bar{P}_{r r}$ respectively denote the trial-averaged power spectrum for

704 stimulus and response time series, and $\left|\bar{P}_{r s}\right|$ denotes the magnitude of the trial-

705 averaged cross-spectrum between stimuli and responses. The value of $\kappa$ for any

706 specific frequency is between 0 and 1 , with larger $\kappa$ indicating stronger phase-locked

707 responses.

708 For a specific variable ( $p$ or $p(1-p)$ ), we computed the phase coherence between

709 stimulus sequence and MEG signals separately for each subject, each magnetometer

710 and each cycle and numerosity condition. In particular, we down-sampled epoched

711 MEG time series to $300 \mathrm{~Hz}$, applied zero padding and Hanning window to single trials, 
712 and used Fast Fourier Transformation (FFT) to calculate power spectrum. The

713 resulting frequency resolution was $0.03 \mathrm{~Hz}$.

714 To evaluate the chance-level phase coherence, we shuffled the MEG time series

715 across different time points within each trial and computed phase coherence for the

716 permutated time series. This permutation procedure was repeated for 500 times to

717 produce a distribution of chance-level phase coherences.

718

719 Decoding Analysis

720 Time-resolved decoding. For an aperiodic stimulus sequence $(p(1-p)$ in $P$-cycle or $p$

721 in U-cycle), we could infer the brain's encoding of the stimulus variable at a specific

722 time lag to stimulus onset by examining how well the value of the variable could be

723 reconstructed from the neural responses at the time lag. In particular, we performed a

724 time-resolved decoding analysis using regression methods [28, 29, 59, 60]:

$$
S(t)=\sum_{n} g(\tau, n) R(t+\tau, n)+\varepsilon(t)
$$

726 where $S(t)$ denotes the value of the stimulus that starts at time $t, g(\tau, n)$ denotes

727 the to-be-estimated decoding weight for channel $n$ at time lag, $R(t+\tau, n)$ denotes

728 the response of channel $n$ at time $t+\tau$, and $\varepsilon(t)$ is a Gaussian noise term.

729 We used the MEG signals of all 306 sensors (102 magnetometers and 204

730 gradiometers) at single time lags to decode $p$ from $U$-cycle trials or $p(1-p)$ from $P$-cycle

731 trials, separately for each subject, each cycle and numerosity condition, and each time

732 lag between 0 and $900 \mathrm{~ms}$. Epoched MEG time series were first down-sampled to 120

$733 \mathrm{~Hz}$. To eliminate multicollinearity and reduce overfitting, we submitted the time series 
734 of the 306 sensors to a principal component analysis (PCA) and used the first 30 components (explaining approximately $95 \%$ variance) as the regressors. The decoding weights (i.e. regression coefficients) were estimated for normalized stimuli and 737 responses using the L2-rectified regression method implemented in the mTRF toolbox 738 [28], with the ridge parameter set to 1. We used a leave-one-out cross-validation [61] to evaluate the predictive power of

740 decoding performance as the following. Out of the 50 trials in question (or 49 trials in 741 the case of trial exclusion), each time one trial served as the test set and the remaining 742 trials as the training set. The decoding weights estimated from the training set were 743 applied to the test set, for which Pearson's $r$ was calculated between the predicted and 744 the ground-truth stimulus sequence. Such computation was repeated for each trial as 745 the test set and the averaged Pearson's $r$ was used as the measure for decoding 746 performance.

We identified time windows that had above-chance decoding performance using 748 cluster-based permutation tests [62] as follows. Adjacent time lags with significantly 749 positive decoding performance at the uncorrected significance level of .05 by right750 sided one-sample $t$ tests were grouped into clusters and the summed $t$-value across 751 the time lags in a cluster was defined as the cluster-level statistic. We randomly 752 shuffled the stimulus sequence of each trial, performed the time-resolved decoding 753 analysis on the shuffled sequence and recorded the maximum cluster-level statistic.

754 This procedure was repeated for 500 times to produce a reference distribution of 755 chance-level maximum cluster-level statistic, based on which we calculated the $P$ 
756 value for each cluster in real data. This test effectively controls the Type I error rate

757 in situations involving multiple comparisons. For each subject, we defined the median

758 of all time points for which the decoding performance exceeded the $95 \%$ percentile of

759 the distribution as the time lag of the peak [63].

760

761 Spatial decoding. For the conditions and time windows that had above-chance

762 performances in the time-resolved decoding analysis, we further performed a spatial

763 decoding analysis at individual sensor location to locate the brain regions that are

764 involved in encoding the variable in question. For a specific senor location, the MEG

765 signals at its three sensors (one magnetometer and two gradiometers) within the time

766 window were used for decoding. The time window that enclosed the peak in the time-

767 resolved decoding was 125-ms and 167-ms wide respectively for $p$ and $p(1-p)$ under

$768 N$-large condition. The decoding procedure was similar to that of the time-resolved

769 decoding described above, except that the number of PCA component used was 19

770 and 24 respectively for decoding $p$ and $p(1-p)$, which explained approximately $99 \%$

771 variance of the original 48 (16 time lags $\times 3$ sensors) and 63 (21 time lags $\times 3$ sensors)

772 channels.

774 Statistical Analysis

775 For phase coherence or decoding performance, grand averages across subjects were

776 computed and permutation tests described above were used to assess their statistical

777 significance over chance [62]. The false discovery rate (FDR) method [64, 65] was 
bioRxiv preprint doi: https://doi.org/10.1101/2019.12.17.879684; this version posted June 1, 2020. The copyright holder for this preprint (which was not certified by peer review) is the author/funder, who has granted bioRxiv a license to display the preprint in perpetuity. It is made available under aCC-BY 4.0 International license.

778 used for multiple comparison corrections whenever applicable. For the phase

779 coherence spectrum averaged across magnetometers, FDR corrections were

780 performed among the 235 frequency bins within $0-7 \mathrm{~Hz}$. For the phase coherence

781 topography at $3.33 \mathrm{~Hz}, F D R$ corrections were performed among all 102

782 magnetometers.

783

784 


\section{References}

786

787

788

789

790

791

792

793

794

795

796

797

798

799

800

801

802

803

804

805

806

807

808

809

810

811

812

813

814

815

816

817

818

819

820

821

822

823

824

1. Zhang $\mathrm{H}$ and Maloney LT, Ubiquitous log odds: a common representation of probability and frequency distortion in perception, action, and cognition. Frontiers in Neuroscience, 2012. 6: 1

2. Attneave F, Psychological probability as a function of experienced frequency. Journal of Experimental Psychology, 1953. 46(2): 81-86

3. Lichtenstein S, Slovic P, Fischhoff B, Layman M, and Combs B, Judge Frequency of Lethal Events. Journal of Experimental Psychology: Human Learning and Memory, 1978. 4(6): 551-578

4. Varey CA, Mellers BA, and Birnbaum MH, Judgments of Proportions. Journal of Experimental Psychology: Human Perception and Performance, 1990. 16(3): 613-625

5. Erev I, Wallsten TS, and Budescu DV, Simultaneous over- and underconfidence: the role of error in judgment processes. Psychological Review, 1994. 101(3): 519-527

6. Gigerenzer G, Hoffrage U, and Kleinbölting H, Probabilistic mental models: a Brunswikian theory of confidence. Psychological Review, 1991. 98(4): 506-528

7. Wallsten TS, Budescu DV, Erev I, and Diederich A, Evaluating and combining subjective probability estimates. Journal of Behavioral Decision Making, 1997. 10: $243-268$

8. Gonzalez R and Wu G, On the Shape of the Probability Weighting Function. Cognitive Psychology, 1999. 38: 129-166

9. Zhang $\mathrm{H}$, Ren X, and Maloney LT, The bounded rationality of probability distortion. bioRxiv [Preprint]. 2019 bioRxiv 662429 [posted 2019 Jun 6; revised 2019 Nov 14; revised 2020 May 23; cited 2020 May 31] Available from:

https://www.biorxiv.org/content/10.1101/662429v3 doi: 10.1101/662429

10. Tversky A and Kahneman D, Advances in Prospect Theory: Cumulative Representation of Uncertainty. Journal of Risk and Uncertainty, 1992. 5: 297323

11. Luce RD, Utility of gains and losses: measurement - theoretical, and experimental approaches, ed. S.W. Link and J.T. Townsend. 2000, Mahwah, New Jersey, London: Lawrence Erlbaum Associates, Inc.

12. Stauffer WR, Lak A, Bossaerts P, and Schultz W, Economic Choices Reveal Probability Distortion in Macaque Monkeys. The Journal of Neuroscience, 2015. 35(7): 3146-3154

13. Ferrari-Toniolo S, Bujold PM, and Schultz W, Probability distortion depends on choice sequence in Rhesus Monkeys. The Journal of Neuroscience, 2019. 39(15): 2915-2929

14. Yang T and Shadlen MN, Probabilistic reasoning by neurons. Nature, 2007. 447: $1075-1080$ 
15. Fennell $\mathrm{J}$ and Baddeley $\mathrm{R}$, Uncertainty plus prior equals rational bias: an intuitive Bayesian probability weighting function. Psychological Review, 2012. 119(4): 878-887

16. Martins ACR, Probability biases as Bayesian inference. Judgment and Decision Making, 2006. 1(2): 108-117

17. See KE and Fox CR, Between ignorance and truth: partition dependence and learning in judgment under uncertainty. Journal of Experimental Psychology: Learning, Memory, and Cognition, 2006. 32(6): 1385-1402

18. Lebreton M, Abitbol R, Daunizeau J, and Pessiglione M, Automatic integration of confidence in the brain valutation signal. Nature Neuroscience, 2015. 18(8): 1159-1167

19. van den Berg R, Awh E, and Ma WJ, Factorial comparison of working memory models. Psychological Review, 2014. 121(1): 124-149

20. Akaike $\mathrm{H}, \mathrm{A}$ new look at the statistical model identification. IEEE Transactions on Automatic Control, 1974. 19(6): 716-723

21. Hurvich CM and Tsai CL, Regression and time series model selection in small samples. Biometrika, 1989. 76(2): 297-307

22. Stephan KE, Penny WD, Daunizeau J, Moran RJ, and Friston KJ, Bayesian model selection for group studies. Neurolmage, 2009. 46: 1004-1017

23. Rigoux L, Stephan KE, Friston KJ, and Daunizeau J, Bayesian model selection for group studies - Revisited. Neurolmage, 2014. 84: 971-985

24. Daunizeau J, Adam V, and Rigoux L, VBA: a probabilistic treatment of nonlinear models for neurobiological and behavioural data. PLoS Computational Biology, 2014. 10(1)

25. Correa CMC, Noorman S, Jiang J, Palminteri S, Cohen MX, Lebreton M, et al., How the level of reward awareness changes the computational and electrophysiological signature of reinforcement learning. The Journal of Neuroscience, 2018. 38(48): 10338-10348

26. Kramer MA and Eden UT, Case studies in neural data analysis: A guide for the practicing neuroscientist. Computational Neuroscience, ed. T.J. Sejnowski and T.A. Poggio. Vol. 11. 2016, the United States of America: Massachusetts Institute of Technology.

27. Lebreton $M$, Bavard S, Daunizeau J, and Palminteri S, Assessing interindividual differences with task-related functional neuroimaging. Nature Human Behaviour, 2019. 3: 897-905

28. Crosse MJ, Liberto GM, Bednar A, and Lalor EC, The Multivariate Temporal Response Function (mTRF) Toolbox: A MATLAB Toolbox for Relating Neural Signals to Continuous Stimuli. frontiers in Human Neuroscience, 2016. 10: 604617

29. Wyart V, Gardelle Vd, Scholl J, and Summerfield C, Rhythmic Fluctuations in Evidence Accumulation during Decision Making in the Human Brain. Neuron, 2012. 76: 847-858

30. Jia J, Liu L, Fang F, and Luo H, Sequential sampling of visual objects during sustained attention. PLOS Biology, 2017. 15(6): 1-19 
869

870

871

872

873

874

875

876

877

878

879

880

881

882

883

884

885

886

887

888

889

890

891

892

893

894

895

896

897

898

899

900

901

902

903

904

905

906

907

908

909

910

31. Liu L, Wang F, Zhou K, Ding N, and Luo H, Perceptual integration rapidly activates dorsal visual pathway to guide local processing in early visual areas. PLoS Biology, 2017. 15(11): e2003646

32. Huang $Q$, Jia J, Han $Q$, and Luo H, Fast-backward replay of sequentially memorized items in humans. eLIFE, 2018. 7: e35164.

33. Snoek L, Miletić S, and Scholte HS, How to control for confounds in decoding analyses of neuroimaging data. Neurolmage, 2019. 184: 741-760

34. Hsu M, Krajbich I, Zhao C, and Camerer CF, Neural Response to Reward Anticipation under Risk Is Nonlinear in Probabilities. The Journal of Neuroscience, 2009. 29(7): 2231-2237

35. Tobler PN, Christopoulos GI, O'Doherty JP, Dolan RJ, and Schultz W, Neuronal Distortions of Reward Probability without Choice. The Journal of Neuroscience, 2008. 28(45): 11703-11711

36. Maloney LT and Zhang H, Decision-theoretic models of visual perception and action. Vision Research, 2010. 50(23): 2362-2374

37. Fiorillo CD, Tobler PN, and Schultz W, Discrete coding of reward probability and uncertainty by dopamine neurons. Science, 2003. 299(5614): 1898-1902

38. Tobler PN, O'Doherty JP, Dolan RJ, and Schultz W, Reward value coding distinct from risk attitude-related uncertainty coding in human reward systems. J Neurophysiol, 2007. 97: 1621-1632

39. Preuschoff K, 't Hart BM, and Einhäuser W, Pupil dilation signals surprise: evidence for noradrenaline's role in decision making. frontiers in Neuroscience, 2011. 5(115): 1-12

40. van Lieshout LLF, Vandenbroucke ARE, Müller NCJ, Cools R, and de Lange $\mathrm{FP}$, Induction and relief of curiosity elicit parietal and frontal activity. The Journal of Neuroscience, 2018. 38(10): 2579-2588

41. Pouget A, Drugowitsch J, and Kepecs A, Confidence and certainty: distinct probabilistic quantities for different goals. Nature Neuroscience, 2016. 19(3): 366-374

42. Gherman S and Philiastides MG, Neural representations of confidence emerge from the process of decision formation during perceptual choices. Neurolmage, 2015. 106: 134-143

43. Gherman S and Philiastides MG, Human VMPFC encodes early signatures of confidence in perceptual decisions. eLIFE, 2018. 7: e38293.

44. Zizlsperger L, Sauvigny T, Händel B, and Haarmeier T, Cortical representations of confidence in a visual perceptual decision. Nature Communications, 2014. 5: 3940

45. Kiani R and Shadlen MN, Representation of confidence associated with a decision by neurons in the parietal cortex. Science, 2009. 324(8): 759-763

46. Bahrami B, Olsen K, Latham PE, Roepstorff A, Rees G, and Frith CD, Optimally interacting minds. Science, 2010. 329: 1081-1085

47. Koriat A, Metacognition: decision-making processes in self-monitoring and self- 
regulation, in The Wiley Blackwell handbook of judgment and decision making, G. Keren and G. Wu, Editors. 2015, Wiley Blackwell: Malden, MA. p. 356-379. 48. Norcia AM, Appelbaum LG, Ales JM, Cottereau BR, and Rossion B, The steady-state visual evoked potential in vision research: $A$ review. Journal of Vision, 2015. 15(6): 1-46

49. Farnsworth D, The Farnsworth-Munsell 100 Hue and Dichotomous Test for Color Vision. Journal of the Optical Society if America, 1943. 33(10): 568-578

50. Brainard DH, The psychophysics toolbox. Spatial Vision, 1997. 10: 433-436

51. Pelli DG, The VideoToolbox software for visual psychophysics: transforming numbers into movies. Spatial Vision, 1997. 10(4): 437-442

52. Aljuhani $\mathrm{KH}$ and $\mathrm{Al}$ turk $\mathrm{LI}$, Modification of the adaptive Nadaraya-Watson kernel regression estimator. Scientific Research and Essays, 2014. 9(22): 966971

53. Nadaraya EA, On Estimating Regression. Theory of Probability and its Applications, 1964. 9(1): 141-142

54. Watson GS, Smooth regression analysis. Sankhyā: The Indian Journal of Statistics, Series A, 1964. 26(4): 359-372

55. Taulu S and Kajola M, Presentation of electromagnetic multichannel data: The signal space separation method. Journal of Applied Physics, 2005. 97: 124905 1-10

56. Taulu S and Simola J, Spatiotemporal signal space separation method for rejecting nearby interference in MEG measurements. Physics in Medicine and Biology, 2006. 51: 1759-1768

57. Oostenveld R, Fries P, Maris E, and Schoffelen J-M, FieldTrip: open source software for advanced analysis of MEG, EEG and invasive electrophysiological data. Computational Intelligence and Neuroscience, 2011. 2011: 1-9

58. Summerfield $\mathrm{C}$ and Mangels JA, Coherent theta-band EEG activity predicts item-context binding during encoding. Neurolmage, 2004. 24: 692-703

59. Gonçalves NR, Whelan R, Foxe JJ, and Lalor EC, Towards obtaining spatiotemporally precise responses to continuous sensory stimuli in humans: A general linear modeling approach to EEG. Neurolmage, 2014. 97: 196-205

60. Lalor EC, Pearlmutter BA, Reilly RB, McDarby G, and Foxe JJ, The VESPA: A method for the rapid estimation of a visual evoked potential. Neurolmage, 2006. 32: 1549-1561

61. Arlot $S$ and Celisse $A, A$ survey of cross-validation procedures for model selection. Statistics Surveys, 2010. 4: 40-79. doi: 10.1214/09-SS054.

62. Maris E and Oostenveld R, Nonparametric statistical testing of EEG- and MEGdata. Journal of Neuroscience Methods, 2007. 164: 177-190

63. Marti S, King J-R, and Dehaene S, Time-resolved decoding of two processing chains during dual-task interference. Neuron, 2015. 88: 1297-1307

64. Benjamini $Y$ and Hochberg $Y$, Controlling the false discovery rate: a practical and powerful approach to multiple testing. Journal of the Royal Statistical Society, 1995. 57(1): 289-300 
bioRxiv preprint doi: https://doi org/10.1101/2019.12.17.879684; this version posted June 1, 2020. The copyright holder for this preprint (which was not certified by peer review) is the author/funder, who has granted bioRxiv a license to display the preprint in perpetuity. It is made available under aCC-BY 4.0 International license.

954

65. Benjamini $Y$ and Yekutieli $D$, The control of the false discovery rate in multiple

955 testing under dependency. The Annals of Statistics, 2001. 29(4): 1165-1188

956 


\section{Supporting Information}

S1 Text. Bounded Log-Odds (BLO) model.

959

S2 Text. Factorial model comparison.

S3 Text. Autocorrelation analysis for periodic and aperiodic stimulus sequences.

S4 Text. Phase coherence and time-resolved decoding analyses for confounding factors.

S5 Text. Explaining the "bumps" in the phase coherence spectra of the confounding factors.

S6 Text. Explaining the higher phase coherence and decoding performance for $p$ or $p(1-p)$ in the $N$-large condition than in the $N$-small condition.

S7 Text. Time-resolved decoding analyses based on cross-validated version of confound regression (CVCR).

S1 Fig. Individual subjects' $\pi(p)-p$ as a function of $p$. The deviation of the subjective from objective relative-frequency, $\pi(p)-p$, is plotted as a function of $p$, separately for the $N$-small (left panel) and $N$-large (right panel) conditions of each subject. Black curves denote smoothed data. Red and blue curves respectively denote BLO and LLO model fits.

S2 Fig. Results of model recovery analysis. Model parameters had been estimated for individual subjects and the 22 subjects' fitted models were used to generate synthetic datasets of 22 virtual subjects. We generated 50 synthetic datasets for each of the 18 models considered in our factorial model comparison analysis. (A) Model identifiability analysis. We fit all the 18 models to each synthetic dataset and identified the best fitting model. The heatmaps represent confusion matrix that quantifies how likely a specific model was correctly identified as the best fitting model for the datasets generated by itself and how likely mistakenly as the best model for the datasets generated by the other models. Each column is for one specific model that was used to generate the datasets. Each row is for one model that was fit to the datasets generated by 18 models. Top: Summed $\triangle \mathrm{AICc}$ was used to identify the best model for each dataset. The color of each cell codes the proportion 
994 that the model on its row was identified as the best model for the 50 datasets generated 995 by the model on its column. Synthetic datasets that were generated by BLO were all 996 best fit by BLO (see the leftmost column). Out of the 850 datasets generated from the 997 other 17 models, only $0.24 \%$ were mis-identified to BLO (see the bottom row). Bottom: 998 The group-level Bayesian model selection was used to quantify the probability for each 999 specific model to be the best model for the dataset. The color of each cell codes the 1000 mean protected exceedance probability of the model on its row had for the 50 datasets 1001 generated by the model on its column. Higher value is coded as more reddish and 1002 lower value as more bluish. Values in each column add up to 1. From left (bottom) to 1003 right (top), the 18 models are 111, 112, 113, 121, 122, 123, 211, 212, 213, 221, 222, $1004223,311,312,313,321,322,323$, where the first digit indexes the D1 assumption (1 1005 for log-odds, 2 for Prelec, and 3 for linear), the second digit indexes the D2 assumption 1006 ( 1 for bounded and 2 for bounds-free), and the third digit indexes the D3 assumption 1007 (1 for $V(\hat{p})$ with $n_{s}=b+N^{a}, 2$ for $V(\hat{p})$ with constant $n_{s}$, and 3 for constant $V(\hat{p})$ ). 1008 The BLO model with sample size $n_{s}=b+N^{a}$ is the first model (111), corresponding 1009 to the leftmost column and the bottom row, which is indicated by an arrow in the plot. 1010 We can see the synthetic data that were generated by BLO were all best by BLO (see 1011 leftmost column) and those generated by the other models were seldom best fit by 1012 BLO (see bottom row). (B) Parameter recovery analysis for BLO. For the $101350 \times 22=1100$ virtual subjects generated by the BLO model with $n_{s}=b+N^{a}$, the 1014 recovered parameters of BLO are plotted against the estimated parameters that were 1015 used to generate synthetic data. Each panel is for one parameter. Each dot is for one 1016 virtual subject. The parameters $\eta, \alpha$ and $b$ that had positively skewed distributions 1017 were transformed into log scale for better visualization. A small proportion of extreme 1018 values $(1.55 \%, 1.55 \%$ and $0.19 \%$, respectively for $\eta, \alpha$ and $b)$ are outside the display 1019 range. The value of $r_{s}$ on each panel indicates Spearman's correlation coefficient between the estimated and recovered parameters.

\section{S3 Fig. Estimated parameters for the BLO model.}

1023 In the box plot, the middle line denotes the median estimate across subjects, the 1024 bottom and top lines respectively denote the lower and upper quartiles, and the error 1025 bars denote the $99 \%$ confidence interval. Dots denote estimates for individual subjects. 1026 The parameters $\eta, a$ and $b$ were transformed into log scale for better visualization.

\section{S4 Fig. Autocorrelations of stimulus sequences as functions of time lags.}

1029 Left: the $P$-cycle condition. Right: the $U$-cycle condition. Solid and dashed lines are 1030 respectively for the $p$ and $p(1-p)$ sequences. The stimulus sequences used for 1031 autocorrelation calculations were from the representative subject whose behavior 1032 results are showed in Fig 2A. All other subjects had similar autocorrelation patterns. 
S5 Fig. Results of control analysis for the confounding variables.

1035 (A) Correlation analysis. Top row: Pearson's correlations between the $p$ sequence and the eight confounding variables $\left(N, N_{\mathrm{t}}, N_{\circ}, A v g L u m i, v C I E-L^{*}, v C I E-a^{*}, v C I E-b^{*}\right.$ and $M$-contrast) separately for the four cycle and numerosity conditions. Bottom row: Pearson's correlations between the $p(1-p)$ sequence and the eight confounding variables for each condition. The results confirmed our design that all of the confounding variables had negligible or moderate correlations with $p$ and $p(1-p)$. (B)

1041 Phase coherence analysis. Grand-averaged phase coherence spectrum between 1042 the sequences of the eight confounding variables and neural responses from 1043 magnetometers. Here we have reproduced the results of the phase coherence 1044 analysis for $p$ and $p(1-p)$ (inside the black frame) to facilitate a comparison between the main analysis and the control analysis. The observed phase coherence spectra of the confounding variables had different patterns than those of $p$ or $p(1-p)$. The vertical line marks $3.33 \mathrm{~Hz}$. See S5 Text and S6 Fig for the possible cause of the "bumps" between 0 and $6.67 \mathrm{~Hz}$. (C) Time-resolved decoding analysis. The same timeresolved decoding procedures as Fig 5 were applied to the eight confounding variables. Here we have reproduced the results of the time-resolved decoding analysis for $p$ and $p(1-p)$ (inside the black frame) to facilitate a comparison between the main analysis and the control analysis. The time-resolved decoding performance of the confounding variables had different patterns than those of $p$ or $p(1-p)$. For $(\mathbf{A})-(\mathbf{C})$, light and dark green respectively code the $N$-small and $N$-large conditions. For (B) and (C), solid and dashed lines respectively denote the $P$-cycle and $U$-cycle conditions. Shadings denote SEM across subjects.

\section{S6 Fig. Explaining the "bumps" in the phase coherence of numerosity.}

Simulated neural signals were generated through convolution of TRF estimated from the MEG2521 sensor (located right occipital) with the $N$ sequence, perturbed by

1061 Gaussian nose whose standard deviation was set to 4 . The simulated neural signals

1062 were then submitted to the phase coherence analysis (see Materials and Methods).

1063 Light and dark green curves denote the phase coherence across magnetometers of 1064 real data from one example subject. Orange curves denote the average across 500 1065 simulations. The "bump" across $0-6.67 \mathrm{~Hz}$ observed in real data was reproduced in 1066 the simulated data. 
1072 S8 Fig. Individual subjects' time-resolved decoding performance for $\boldsymbol{p}$ (left 1073 column) and $p(1-p)$ (right column), separately for the $\mathbf{N}$-small (top row) and $\mathbf{N}$ 1074 large (bottom row) conditions.

1075 Each row on the heatmap is for one subject. Higher level of decoding performance is coded as more reddish and lower level as more bluish. The green curve superimposed on the heatmap denotes grand-averaged decoding performance across subjects (see the right $y$-axis). Symbols on the top of each panel indicate time lags that had abovechance decoding performance (cluster-based permutation tests), with vertical bars representing $P_{\text {cluster }}<0.01$ and dots representing $0.01 \leq P_{\text {cluster }}<0.05$.

S9 Fig. Time-resolved decoding analysis based on cross-validated version of confound regression (CVCR).

1084 (A) Decoding performance for $p(1-p)$. (B) Decoding performance for $V(\hat{p})$. The $V(\hat{p})$ 1085 was computed according to each subject's fitted BLO model (with sample size $n_{s}=$ $\left.1086 b+N^{a}\right)$. Curves denote grand-averaged decoding performance over different time 1087 lags, separately for the $\mathrm{N}$-small (light green) and $\mathrm{N}$-large (dark green) conditions. 1088 Shadings denote SEM across subjects. Vertical bars above the curves indicate time 1089 lags that had above-chance decoding performance (cluster-based permutation tests, $\left.1090 P_{\text {cluster }}<0.01\right)$. The eight confounding variables we considered in S4 Text $\left(N, N_{t}, N_{o}\right.$, 1091 AvgLumi, vCIE- $L^{*}, v C I E-a^{*}, v C I E-b^{*}$ and $M$-contrast) were regressed out from MEG 1092 time series before decoding analysis. See S7 Text for methodological details. The 1093 decoded time course from CVCR for $p(1-p)$ was similar to that of the standard time1094 resolved decoding analysis (Fig 5, right panel). The decoded time course was also 1095 similar for $V(\hat{p})$.

S10 Fig. Consequences of random sampling errors.

1098 The $p$ or $p(1-p)$ estimated from a sample of dots from a display might deviate from the 1099 true value of $p$ or $p(1-p)$ in the display. As an evaluation of the fidelity of the sampled 1100 value, we used numerical simulations to compute the correlation (Pearson's $r$ ) between 1101 the true value and the sampled value, based on the BLO parameters estimated from 1102 individual subjects. Triangles and circles respectively denote $p$ and $p(1-p)$. The truth1103 sample correlation was higher in the $N$-large condition than in the $N$-small condition. 